\title{
Attractor of Cantor Type with Positive Measure
}

\author{
Janusz Morawiec® and Thomas Zürcher
}

\begin{abstract}
We construct an iterated function system consisting of strictly increasing contractions $f, g:[0,1] \rightarrow[0,1]$ with $f([0,1]) \cap g([0,1])=\emptyset$ and such that its attractor has positive Lebesgue measure.
\end{abstract}

Mathematics Subject Classification. Primary 28A80; Secondary 26A18.

Keywords. Iterated function system, attractor, Cantor type set.

\section{Introduction}

Self-similar measures associated with iterated function systems (shortly IFS) have many significant and interesting applications in various areas of science, including mathematics, and in particular, the theory of functional equations (see e.g. $[1,8,10]$ ). Studying a functional equation connected with the problem posed in $[7,9]$, we come to the following question.

Question 1.1. Consider an IFS consisting of strictly monotone contractions $f_{1}, \ldots, f_{N}:[0,1] \rightarrow[0,1]$ such that

$$
\bigcup_{n=1}^{N} f_{n}([0,1]) \neq[0,1] \quad \text { and } \quad f_{i}((0,1)) \cap f_{j}((0,1))=\emptyset \quad \text { for all } i \neq j .
$$

Is the attractor of this IFS necessary of Lebesgue measure zero?

Surprisingly we could not find any answer to this question by looking through the literature in this topic. The purpose of this paper is to give a negative answer to this question by constructing an example of an IFS consisting of two strictly increasing contractions $f, g:[0,1] \rightarrow[0,1]$ such that $f([0,1]) \cap g([0,1])=\emptyset$ with the attractor of positive Lebesgue measure. 


\section{Preliminaries}

We say that a function $f:[a, b] \rightarrow \mathbb{R}$ is L-Lipschitz, if

$$
|f(x)-f(y)| \leq L|x-y| \text { for all } x, y \in[a, b] .
$$

Any $L$-Lipschitz function $f:[a, b] \rightarrow \mathbb{R}$ with $L<1$ is said to be a contraction.

The following fact will be used frequently in the announced construction; its proof is simple, so we omit it.

Lemma 2.1. Let $f:[a, b] \rightarrow \mathbb{R}$ be a function and let $c \in[a, b]$. If the restrictions of $f$ to $[a, c]$ and to $[c, b]$ are both L-Lipschitz, then $f$ is L-Lipschitz as well.

In this paper, whenever we are given a finite collection of contractions defined on the interval $[0,1]$ into itself, we refer to it as iterated function system.

The following fact is well-known (see [6, Theorem 9.1]).

Theorem 2.2. If $\left\{f_{1}, \ldots, f_{N}\right\}$ is an IFS, then there is a unique attractor, i.e. a non-empty compact set $A_{*} \subset \mathbb{R}$ such that

$$
A_{*}=\bigcup_{n=1}^{N} f_{n}\left(A_{*}\right) \text {. }
$$

Moreover, if $A_{1}=[0,1]$ and $A_{k+1}=\bigcup_{n=1}^{N} f_{n}\left(A_{k}\right)$ for every $k \in \mathbb{N}$, then

$$
A_{*}=\bigcap_{k \in \mathbb{N}} A_{k} .
$$

From the moreover part of Theorem 2.2 we see that the attractor of the IFS considered in Question 1.1 looks like a set of Cantor type; in fact, (1.1) and the strict monotonicity of $f_{1}, \ldots, f_{N}$ imply $A_{k+1} \varsubsetneqq A_{k}$ for every $k \in \mathbb{N}$. Let us mention here that not every set of Cantor type can be an attractor of some IFS (see [2]), and moreover, that typical closed sets in $[0,1]$ can not be attractors of any IFS (see [4]). In particular, for each $s \in(0,1]$ it is possible to construct a nowhere dense perfect subset of $[0,1]$, with Hausdorff dimension $s$, which is not an attractor for any IFS composed of weak contractions of $[0,1]$ into itself (see [3]). It is also known that the family of all attractors is dense, path connected and an $F_{\sigma}$ set in the space of all nonempty and compact subsets of $[0,1]$ endowed with the Hausdorff metric (see [5]).

Note that the strict monotonicity in Question 1.1 is crucial. Indeed, if we omit the word "strictly", then there is no problem to give an example of an IFS whose attractor is of positive Lebesgue measure.

Example 2.3. Define $F, G:[0,1] \rightarrow[0,1]$ by

$$
F(x)=\left\{\begin{array}{ll}
\frac{1}{2} x, & \text { if } x \in\left[0, \frac{1}{3}\right] \\
\frac{1}{6}, & \text { if } x \in\left(\frac{1}{3}, \frac{2}{3}\right) \\
\frac{1}{2} x-\frac{1}{6}, & \text { if } x \in\left[\frac{2}{3}, 1\right]
\end{array} \text { and } \quad G(x)= \begin{cases}\frac{2}{3}+\frac{1}{2} x, & \text { if } x \in\left[0, \frac{1}{3}\right] \\
\frac{5}{6}, & \text { if } x \in\left(\frac{1}{3}, \frac{2}{3}\right) \\
\frac{1}{2} x+\frac{1}{2}, & \text { if } x \in\left[\frac{2}{3}, 1\right] .\end{cases}\right.
$$


A short calculation shows that $F\left(\left[0, \frac{1}{3}\right] \cup\left[\frac{2}{3}, 1\right]\right) \cup G\left(\left[0, \frac{1}{3}\right] \cup\left[\frac{2}{3}, 1\right]\right)=\left[0, \frac{1}{3}\right] \cup\left[\frac{2}{3}, 1\right]$. By Theorem 2.2 the set $\left[0, \frac{1}{3}\right] \cup\left[\frac{2}{3}, 1\right]$ is the attractor of the considered IFS, which clearly is not of Cantor type.

\section{The Similitudes Case}

Now we prove that if the considered IFS consists of similitudes, then the answer to the posed question is positive.

From now on, we denote by $\mathcal{L}^{1}$ the Lebesgue measure on the real line.

Proposition 3.1. Assume that $f_{1}, \ldots, f_{N}:[0,1] \rightarrow[0,1]$ is an IFS consisting of similitudes satisfying (1.1). Then the attractor of this IFS is of Lebesgue measure zero.

Proof. For every $n \in\{1, \ldots, N\}$, let the similitude $f_{n}$ be of the form

$$
f_{n}(x)=a_{n} x+b_{n}
$$

with some $a_{n} \in(-1,0) \cup(0,1)$ and $b_{n} \in[0,1]$.

Put $q=\mathcal{L}^{1}\left(A_{1} \backslash A_{2}\right)$ and observe that $1-q=\sum_{n=1}^{N}\left|a_{n}\right| \in(0,1)$, by (1.1). A simple induction gives $\mathcal{L}^{1}\left(A_{k} \backslash A_{k+1}\right)=q(1-q)^{k-1}$ for every $k \in \mathbb{N}$. Hence

$$
\mathcal{L}^{1}\left(A_{*}\right)=1-\sum_{k=1}^{\infty} \mathcal{L}^{1}\left(A_{k} \backslash A_{k+1}\right)=1-\frac{q}{1-(1-q)}=0,
$$

and the proof is complete.

\section{Construction of the Example}

We begin with an explanation of the idea how we construct the announced example. Consider the IFS consisting of the contractions $f_{0}, g_{0}:[0,1] \rightarrow[0,1]$ defined by

$$
f_{0}(x)=\frac{1}{3} x \quad \text { and } \quad g_{0}(x)=\frac{1}{3} x+\frac{2}{3} .
$$

It is well-known that the attractor of this IFS is the Cantor set (see e.g. [6, Chapter 9]), which has Lebesgue measure zero. The problem is that the gap $\left(\frac{1}{3}, \frac{2}{3}\right)$ leads to the gaps $\left(\frac{1}{9}, \frac{2}{9}\right)$ and $\left(\frac{7}{9}, \frac{8}{9}\right)$. During the process, the gaps propagate and at the end sum up to a set of Lebesgue measure 1 . To counteract, we modify the functions $f_{0}$ and $g_{0}$. As $\left(\frac{1}{3}, \frac{2}{3}\right)$ and its images generate gaps, we make the gaps smaller by mapping $\left(\frac{1}{3}, \frac{2}{3}\right)$ to smaller sets than $\left(\frac{1}{9}, \frac{2}{9}\right)$ and $\left(\frac{7}{9}, \frac{8}{9}\right)$. We continue to modify the functions $f_{0}$ and $g_{0}$ such that the images of the smaller gaps are even smaller. This way, we obtain two sequences of strictly increasing contractions that converge uniformly to strictly increasing contractions that form our IFS. 


\subsection{Key Sequences}

First, we need two sequences $\left(\varepsilon_{k}\right)_{k \in \mathbb{N}}$ and $\left(w_{k}\right)_{k \in \mathbb{N}}$ of parameters that will determine how we modify the functions $f_{0}$ and $g_{0}$.

We let

$$
w_{1}=1 \quad \text { and } \quad \varepsilon_{1}=\frac{1}{6} .
$$

Having defined $\varepsilon_{l}>0$ and $w_{l} \in \mathbb{R}$ for all $1 \leq l \leq k$, we let

$$
w_{k+1}=\frac{w_{k}}{2}-\varepsilon_{k}
$$

and choose $\varepsilon_{k+1}>0$ such that the following conditions are satisfied

$$
\begin{aligned}
2^{k} \varepsilon_{k+1} & <\frac{1}{2} \cdot \frac{1}{4^{k}}, \\
\frac{\varepsilon_{k+1}}{\varepsilon_{k}} & <\frac{1}{2}, \\
\varepsilon_{k+1} & <\frac{w_{k}}{4}-\frac{\varepsilon_{k}}{2} .
\end{aligned}
$$

To see that the sequences $\left(\varepsilon_{k}\right)_{k \in \mathbb{N}}$ and $\left(w_{k}\right)_{k \in \mathbb{N}}$ are well-defined, we only have to show that we really can satisfy (4.6). First, we observe that $\frac{w_{1}}{4}-\frac{\varepsilon_{1}}{2}=$ $\frac{1}{4}-\frac{1}{12}>0$. Thus we can choose $\varepsilon_{2}$. Fix $k \in \mathbb{N}$ and assume that we have already chosen $\varepsilon_{k+1}$ and $w_{k+1}$. Then, using (4.3) and (4.6), we have $\frac{w_{k+1}}{4}-\frac{\varepsilon_{k+1}}{2}=$ $\frac{w_{k}}{8}-\frac{\varepsilon_{k}}{4}-\frac{\varepsilon_{k+1}}{2}>0$, which shows that we can choose $\varepsilon_{k+2}$.

Condition (4.4) will be used to show that the attractor of the constructed IFS has positive Lebesgue measure. To guarantee that our functions are contractions, we will need condition (4.5). Finally, conditions (4.6) and (4.3) will guarantee that all the intervals where the modifications will take place are non-degenerated but small enough.

Lemma 4.1. For every $k \in \mathbb{N}$ we have

$$
0<w_{k} \leq \frac{1}{2^{k-1}}
$$

and

$$
\frac{w_{k+1}}{w_{k}}<\frac{1}{2} .
$$

Proof. Conditions (4.7) and (4.8) are clearly true for $k=1$.

If $k \geq 2$, then using (4.3) and (4.6), we get $w_{k+1}=\frac{w_{k}}{2}-\varepsilon_{k}>2 \varepsilon_{k+1}>0$. Thus the first inequality in (4.7) is proved. To prove the second one and (4.8), it is enough to observe that applying (4.3) we have $w_{k+1}=\frac{w_{k}}{2}-\varepsilon_{k}<\frac{w_{k}}{2}$ and proceed by induction on $k$. 


\subsection{Intervals Where the Modifications Take Place}

We inductively define a sequence of collections of intervals as follows. Put

$$
\mathcal{I}_{1}=\{[0,1]\}
$$

and observe that the only interval in $\mathcal{I}_{1}$ has length $w_{1}$.

Fix $k \in \mathbb{N}$ and assume that the collection $\mathcal{I}_{k}$ has been defined in such a way that $b-a=w_{k}$ for each $[a, b] \in \mathcal{I}_{k}$; note that $w_{k}>0$ by (4.7). Next observe that if $[a, b] \in \mathcal{I}_{k}$, then according to (4.3) we have

$\frac{a+b}{2}-\varepsilon_{k}-a=\frac{w_{k}}{2}-\varepsilon_{k}=w_{k+1} \quad$ and $\quad b-\frac{a+b}{2}-\varepsilon_{k}=\frac{w_{k}}{2}-\varepsilon_{k}=w_{k+1}$.

Now we put

$$
\mathcal{I}_{k+1}=\bigcup_{[a, b] \in \mathcal{I}_{k}}\left\{\left[a, \frac{a+b}{2}-\varepsilon_{k}\right],\left[\frac{a+b}{2}+\varepsilon_{k}, b\right]\right\} .
$$

In this way we have constructed a sequence $\left(\mathcal{I}_{k}\right)_{k \in \mathbb{N}}$ of collections of intervals. Let us write down some of the sequence's properties in the next lemma.

Lemma 4.2. Assume that $k \in \mathbb{N}$.

(i) The family $\mathcal{I}_{k}$ consists of $2^{k-1}$ pairwise disjoint closed subintervals of $[0,1]$.

(ii) If $[a, b] \in \mathcal{I}_{k}$, then $b-a=w_{k}$.

(iii) We have $\bigcup \mathcal{I}_{k+1} \varsubsetneqq \bigcup \mathcal{I}_{k}$.

(iv) Let $[a, b] \in \mathcal{I}_{k}$. If $[a, b] \subset\left[0, \frac{1}{3}\right]$, then $[a, b]+\frac{2}{3} \in \mathcal{I}_{k}$, and if $[a, b] \subset\left[\frac{2}{3}, 1\right]$, then $[a, b]-\frac{2}{3} \in \mathcal{I}_{k}$.

Proof. Assertions (i), (ii) and (iii) are clear from the construction of the sequence $\left(\mathcal{I}_{k}\right)_{k \in \mathbb{N}}$. Assertion (iv) can be proved by a simple induction with the fact that

$$
\mathcal{I}_{2}=\left\{\left[0, \frac{1}{3}\right],\left[\frac{2}{3}, 1\right]\right\}
$$

as its first step.

\subsection{Attractor}

We now define a set, which turns out to be the attractor of our IFS. For every $k \in \mathbb{N}$ we let

$$
A_{k}=\bigcup \mathcal{I}_{k}
$$

and observe that by assertion (iii) of Lemma 4.2 we have

$$
A_{k+1} \varsubsetneqq A_{k} \text {. }
$$

Now we define $A_{*}$ as intersection of all $A_{k}$ as in (2.1).

It is clear that

$$
A_{*} \subset\left[0, \frac{1}{3}\right] \cup\left[\frac{2}{3}, 1\right] .
$$


Moreover, assertion (iv) of Lemma 4.2 yields

$$
\left(A_{*} \cap\left[0, \frac{1}{3}\right]\right)+\frac{2}{3}=A_{*} \cap\left[\frac{2}{3}, 1\right] .
$$

Lemma 4.3. The set $A_{*}$ is of Cantor type, i.e. nonempty, compact, perfect and nowhere dense.

Proof. It is easy to see that $0 \in A_{*}$, so $A_{*} \neq \emptyset$.

From assertion (i) of Lemma 4.2 we conclude that each $A_{k}$ is closed and bounded. Hence $A_{*}$ is compact.

For showing that $A_{*}$ is nowhere dense, suppose the contrary and choose a point $x_{0} \in A_{*}$ and $r>0$ such that $\left(x_{0}-r, x_{0}+r\right) \subset A_{*}$. Thus $\left(x_{0}-r, x_{0}+r\right) \subset$ $A_{k}$ for every $k \in \mathbb{N}$, which is impossible by assertions (i) and (ii) of Lemma 4.2 and (4.7).

Lemma 4.4. The set $A_{*}$ has positive (one-dimensional) Lebesgue measure.

Proof. By Lemma 4.3 the set $A_{*}$ is Lebesgue measurable. We calculate the measure of the complement of $A^{*}$. In the course of the computation, we need (4.9), assertion (i) of Lemma 4.2 and (4.4), as well as

$$
[0,1] \backslash A_{k} \backslash\left(\bigcup_{l=1}^{k-1}\left([0,1] \backslash A_{l}\right)\right)=A_{k-1} \backslash A_{k} .
$$

Consequently,

$$
\begin{aligned}
\mathcal{L}^{1}\left([0,1] \backslash A_{*}\right) & =\mathcal{L}^{1}\left([0,1] \backslash \bigcap_{k=2}^{\infty} A_{k}\right)=\mathcal{L}^{1}\left(\bigcup_{k=2}^{\infty}\left([0,1] \backslash A_{k}\right)\right) \\
& =\mathcal{L}^{1}\left(\bigcup_{k=2}^{\infty}\left(\left([0,1] \backslash A_{k}\right) \backslash\left(\bigcup_{l=1}^{k-1}\left([0,1] \backslash A_{l}\right)\right)\right)\right) \\
& \leq \sum_{k=2}^{\infty} \mathcal{L}^{1}\left(\left([0,1] \backslash A_{k}\right) \backslash\left(\bigcup_{l=1}^{k-1}\left([0,1] \backslash A_{l}\right)\right)\right) \\
& =\mathcal{L}^{1}\left([0,1] \backslash A_{2}\right)+\sum_{k=3}^{\infty} \mathcal{L}^{1}\left(\left([0,1] \backslash A_{k}\right) \backslash\left(\bigcup_{l=1}^{k-1}\left([0,1] \backslash A_{l}\right)\right)\right) \\
& =2 \varepsilon_{1}+\sum_{k=3}^{\infty} 2^{k-2} \cdot 2 \varepsilon_{k-1}<\frac{1}{3}+\sum_{k=3}^{\infty} \frac{1}{4^{k-2}}=\frac{2}{3} .
\end{aligned}
$$

Finally, we have $\mathcal{L}^{1}\left(A_{*}\right) \geq \frac{1}{3}>0$. 

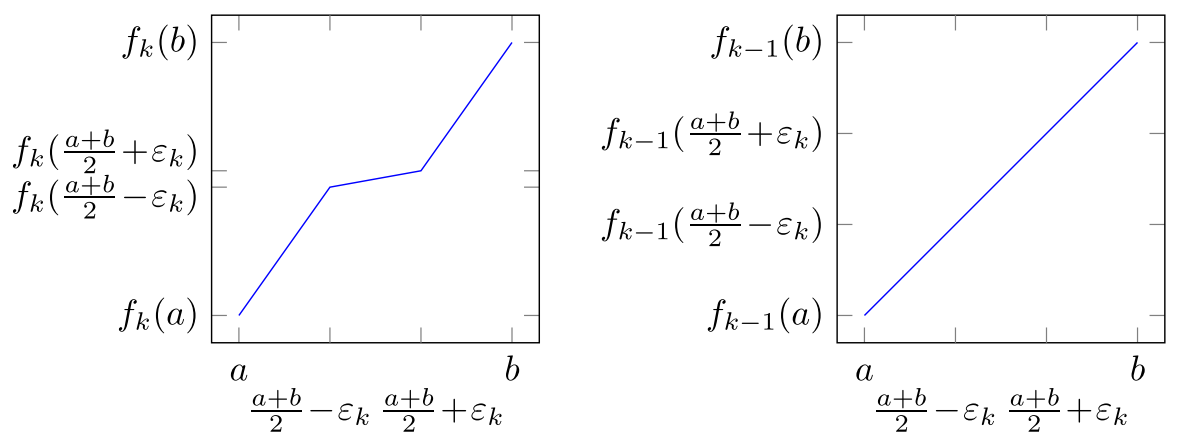

Figure 1. Graphs of $f_{k}$ and $f_{k-1}$ on $[a, b] \in \mathcal{I}_{k}$

\subsection{Sequence of Functions}

Define the function $f_{1}:[0,1] \rightarrow[0,1]$ by

$$
f_{1}(x)= \begin{cases}3\left(\frac{1}{6}-\varepsilon_{2}\right) x, & \text { if } x \in\left[0, \frac{1}{3}\right] \\ 6 \varepsilon_{2}\left(x-\frac{1}{3}\right)+\frac{1}{6}-\varepsilon_{2}, & \text { if } x \in\left(\frac{1}{3}, \frac{2}{3}\right) \\ 3\left(\frac{1}{6}-\varepsilon_{2}\right)\left(x-\frac{2}{3}\right)+\frac{1}{6}+\varepsilon_{2}, & \text { if } x \in\left[\frac{2}{3}, 1\right]\end{cases}
$$

Note that $f_{1}$ is a strictly increasing contraction with the minimal Lipschitz constant strictly smaller than $\frac{1}{2}$; here we use that $\varepsilon_{2} \in\left(0, \frac{1}{16}\right)$ by $(4.4)$ and apply Lemma 2.1. Moreover, simple calculations (some of them with the use of (4.3)) give

$$
f_{1}(0)=f_{0}(0), \quad f_{1}(1)=f_{0}(1), \quad f_{1}\left(\frac{1}{3}\right)-f_{1}(0)=f_{1}(1)-f_{1}\left(\frac{2}{3}\right)=w_{3} .
$$

Fix $k \in \mathbb{N}$ and assume that the function $f_{k-1}:[0,1] \rightarrow[0,1]$ has been defined. Then we define $f_{k}:[0,1] \rightarrow[0,1]$ as follows. If $[a, b] \in \mathcal{I}_{k}$, then we define $f_{k}$ on $[a, b]$ by

$f_{k}(x)= \begin{cases}\frac{w_{k+2}}{w_{k+1}}(x-a)+f_{k-1}(a), & \text { if } x \in\left[a, \frac{a+b}{2}-\varepsilon_{k}\right] \\ \frac{\varepsilon_{k+1}}{\varepsilon_{k}}\left(x-\frac{a+b}{2}+\varepsilon_{k}\right)+f_{k-1}(a)+w_{k+2}, & \text { if }\left|x-\frac{a+b}{2}\right|<\varepsilon_{k} \\ \frac{w_{k+2}}{w_{k+1}}\left(x-\frac{a+b}{2}-\varepsilon_{k}\right)+f_{k-1}(a)+w_{k+2}+2 \varepsilon_{k+1}, & \text { if } x \in\left[\frac{a+b}{2}+\varepsilon_{k}, b\right]\end{cases}$

see Fig. 1. Note that the above formula is consistent with the definition of $f_{1}$ by (4.3) and simple calculations. In this way we have defined $f_{k}$ on $\bigcup \mathcal{I}_{k}$. Now we put

$$
f_{k}(x)=f_{k-1}(x) \text { for every } x \in[0,1] \backslash \bigcup \mathcal{I}_{k} .
$$

The next lemma collects essential properties of the just defined sequence $\left(f_{k}\right)_{k \in \mathbb{N}}$.

Lemma 4.5. Assume that $k \in \mathbb{N}$.

(i) If $[a, b] \in \mathcal{I}_{k+1}$, then $f_{k}(b)-f_{k}(a)=w_{k+2}$. 
(ii) If $[a, b] \in \mathcal{I}_{k}$, then $f_{k}(a)=f_{k-1}(a)$ and $f_{k}(b)=f_{k-1}(b)$.

(iii) The function $f_{k-1}$ is affine on each $[a, b] \in \mathcal{I}_{k}$.

(iv) The function $f_{k}$ is strictly increasing.

(v) The function $f_{k}$ is a contraction with Lipschitz constant strictly smaller than $\frac{1}{2}$.

(vi) We have $f_{k}([0,1])=\left[0, \frac{1}{3}\right]$.

(vii) If $[a, b] \in \mathcal{I}_{k}$, then $\left[f_{k-1}(a), f_{k-1}(b)\right] \in \mathcal{I}_{k+1}$.

(viii) If $[a, b] \in \mathcal{I}_{k+1}$, then there exists an interval $[c, d] \in \mathcal{I}_{k}$ such that either $[a, b]=\left[f_{k-1}(c), f_{k-1}(d)\right]$ or $[a, b]=\left[f_{k-1}(c), f_{k-1}(d)\right]+\frac{2}{3}$.

Proof. (i) Fix an interval $[c, d] \in \mathcal{I}_{k+1}$. Then there exists an interval $[a, b] \in$ $\mathcal{I}_{k}$ such that either $[c, d]=\left[a, \frac{a+b}{2}-\varepsilon_{k}\right]$ or $[c, d]=\left[\frac{a+b}{2}+\varepsilon_{k}, b\right]$.

If $[c, d]=\left[a, \frac{a+b}{2}-\varepsilon_{k}\right]$, then using assertion (ii) of Lemma 4.2 and (4.3) we obtain

$$
\begin{aligned}
f_{k}\left(\frac{a+b}{2}-\varepsilon_{k}\right) & =\frac{w_{k+2}}{w_{k+1}}\left(\frac{b-a}{2}-\varepsilon_{k}\right)+f_{k-1}(a) \\
& =\frac{w_{k+2}}{w_{k+1}}\left(\frac{w_{k}}{2}-\varepsilon_{k}\right)+f_{k-1}(a)=w_{k+2}+f_{k-1}(a) .
\end{aligned}
$$

Hence $f_{k}(d)-f_{k}(c)=w_{k+2}$.

If $[c, d]=\left[\frac{a+b}{2}+\varepsilon_{k+1}, b\right]$, then the same arguments as above give

$$
f_{k}(d)-f_{k}(c)=f_{k}(b)-f_{k}\left(\frac{a+b}{2}+\varepsilon_{k}\right)=\frac{w_{k+2}}{w_{k+1}}\left(\frac{b-a}{2}-\varepsilon_{k}\right)=w_{k+2} \text {. }
$$

(ii) Fix $[a, b] \in \mathcal{I}_{k}$. We see at once that $f_{k}(a)=f_{k-1}(a)$. Applying assertion (ii) of Lemma 4.2, the just proven assertion (i) and (4.3), we obtain

$$
\begin{aligned}
f_{k}(b) & =\frac{w_{k+2}}{w_{k+1}}\left(\frac{b-a}{2}-\varepsilon_{k}\right)+f_{k-1}(a)+w_{k+2}+2 \varepsilon_{k+1} \\
& =\frac{w_{k+2}}{w_{k+1}}\left(\frac{w_{k}}{2}-\varepsilon_{k}\right)+f_{k-1}(b)-w_{k+1}+w_{k+2}+2 \varepsilon_{k+1} \\
& =2 w_{k+2}+f_{k-1}(b)-w_{k+1}+2 \varepsilon_{k+1}=f_{k-1}(b) .
\end{aligned}
$$

(iii) Clearly, $f_{0}$ is affine on $[0,1]$. Then a simple induction completes the proof.

(iv) It is enough to observe that on each interval $[a, b] \in \mathcal{I}_{k}$ the function $f_{k}$ is strictly increasing by (4.7), and then proceed by induction on $k$ using assertion (ii).

(v) We first observe that $f_{k}$ is continuous on any interval $[a, b] \in \mathcal{I}_{k}$; continuity at $\frac{a+b}{2}+\varepsilon_{k}$ is clear and continuity at $\frac{a+b}{2}-\varepsilon_{k}$ follows from (4.10). Then combining (4.8) with (4.5) and applying Lemma 2.1 jointly with assertion (ii) we conclude that $f_{k}$ restricted to any interval $[a, b] \in \mathcal{I}_{k}$ is $L$-Lipschitz with $L<\frac{1}{2}$. Now the assertion can be proved by induction on $k$.

(vi) From the construction we can easy conclude, proceeding by induction with the use of assertion (ii), that for every $k \in \mathbb{N}$ we have $f_{k}(0)=0$ and $f_{k}(1)=\frac{1}{3}$. 
(vii) The assertion is clear for $k=1$.

Fix $k \in \mathbb{N}$ and assume inductively that (vii) holds. Fix also an interval $[c, d] \in \mathcal{I}_{k+1}$. Then there exists an interval $[a, b] \in \mathcal{I}_{k}$ such that either $[c, d]=\left[a, \frac{a+b}{2}-\varepsilon_{k}\right]$ or $[c, d]=\left[\frac{a+b}{2}+\varepsilon_{k}, b\right]$.

First, we consider the case where $[c, d]=\left[a, \frac{a+b}{2}-\varepsilon_{k}\right]$. Assertion (ii) gives $f_{k}(c)=f_{k}(a)=f_{k-1}(a)$. Then assertion (i) and the induction hypothesis imply $\left[f_{k}(c), f_{k}(c)+w_{k+1}\right]=\left[f_{k-1}(a), f_{k-1}(a)+w_{k+1}\right]=$ $\left[f_{k-1}(a), f_{k-1}(b)\right] \in \mathcal{I}_{k+1}$. Hence, by the definition of $\mathcal{I}_{k+2}$, we see that $\left[f_{k}(c), f_{k}(c)+\frac{w_{k+1}}{2}-\varepsilon_{k+1}\right] \in \mathcal{I}_{k+2}$. Finally, according to assertion (i) and (4.3) we conclude that

$\left[f_{k}(c), f_{k}(d)\right]=\left[f_{k}(c), f_{k}(c)+w_{k+2}\right]=\left[f_{k}(c), f_{k}(c)+\frac{w_{k+1}}{2}-\varepsilon_{k+1}\right] \in \mathcal{I}_{k+2}$.

Now, we consider the case where $[c, d]=\left[\frac{a+b}{2}+\varepsilon_{k}, b\right]$. Assertion (ii) gives $f_{k}(d)=f_{k}(b)=f_{k-1}(b)$. Then assertion (i) and the induction hypothesis imply $\left[f_{k}(d)-w_{k+1}, f_{k}(d)\right]=\left[f_{k-1}(b)-w_{k+1}, f_{k-1}(b)\right]=$ $\left[f_{k-1}(a), f_{k-1}(b)\right] \in \mathcal{I}_{k+1}$. Hence, by the definition of $\mathcal{I}_{k+2}$, we see that $\left[f_{k}(d)-\frac{w_{k+1}}{2}+\varepsilon_{k+1}, f_{k}(d)\right] \in \mathcal{I}_{k+2}$. Finally, according to assertion (i) and (4.3) we conclude that

$$
\left[f_{k}(c), f_{k}(d)\right]=\left[f_{k}(d)-w_{k+2}, f_{k}(d)\right]=\left[f_{k}(d)-\frac{w_{k+1}}{2}+\varepsilon_{k+1}, f_{k}(d)\right] \in \mathcal{I}_{k+2} .
$$

(viii) From assertion (i) of Lemma 4.2 we see that the families $\mathcal{I}_{k}$ and $\mathcal{I}_{k+1}$ consist of $2^{k-1}$ and $2^{k}$ pairwise disjoint closed intervals, respectively. The just proved assertion (vii) and assertion (iv) of Lemma 4.2 imply that with each interval $[a, b] \in \mathcal{I}_{k}$ there are associated exactly two intervals of the form $\left[f_{k-1}(a), f_{k-1}(b)\right]$ and $\left[f_{k-1}(a), f_{k-1}(b)\right]+\frac{2}{3}$, and both belong to $\mathcal{I}_{k+1}$. It remains to note that if $[a, b]$ and $[c, d]$ are different intervals from $\mathcal{I}_{k}$, then the intervals associated with them are pairwise disjoint, by assertions (iv) and (vi).

The proof is complete.

\subsection{The Limit Function}

We show that the sequence $\left(f_{k}\right)_{k \in \mathbb{N}}$ converges pointwise to a strictly increasing contraction. We begin with showing that it is convergent.

Lemma 4.6. The sequence $\left(f_{k}\right)_{k \in \mathbb{N}}$ is a Cauchy sequence with respect to the supremum norm.

Proof. Fix $k \in \mathbb{N}$, interval $[a, b] \in \mathcal{I}_{k}$ and note that we only need to show that $\sup \left\{\left|f_{k}(x)-f_{k-1}(x)\right|: x \in[a, b]\right\} \leq \frac{1}{2^{k}}$. According to assertion (ii) of Lemma 4.5 the supremum is attained at the point $\frac{a+b}{2}-\varepsilon_{k}$ (see Fig. 1). Therefore, it suffices to prove that $M=\left|f_{k}\left(\frac{a+b}{2}-\varepsilon_{k}\right)-f_{k-1}\left(\frac{a+b}{2}-\varepsilon_{k}\right)\right| \leq \frac{1}{2^{k}}$. 
Making use of (4.10), assertions (iii) and (i) of Lemma 4.5, assertion (ii) of Lemma 4.2, (4.8), (4.3), and Lemma 4.1 we get

$$
\begin{aligned}
M & =\left|w_{k+2}+f_{k-1}(a)-f_{k-1}\left(\frac{a+b}{2}-\varepsilon_{k}\right)\right| \\
& =\left|w_{k+2}+f_{k-1}(a)-\frac{f_{k-1}(b)-f_{k-1}(a)}{b-a}\left(\frac{a+b}{2}-\varepsilon_{k}-a\right)-f_{k-1}(a)\right| \\
& =\left|w_{k+2}-\frac{w_{k+1}}{w_{k}}\left(\frac{w_{k}}{2}-\varepsilon_{k}\right)\right| \leq w_{k+2}+\frac{1}{2} w_{k+1} \leq \frac{1}{2^{k}} .
\end{aligned}
$$

The proof is complete.

Define the function $f:[0,1] \rightarrow[0,1]$ by

$$
f(x)=\lim _{k \rightarrow \infty} f_{k}(x) ;
$$

Lemma 4.6 shows that $f$ is well-defined and continuous.

Lemma 4.7. The function $f$ is strictly increasing.

Proof. The function $f$ is increasing by assertion (iv) of Lemma 4.5.

Suppose the assertion of the lemma is false. Then there exists an interval $[x, y] \subset[0,1]$ on which $f$ is constant.

By Lemma 4.3, the set $A_{*}$ is closed and nowhere dense. Hence, we find a point $z \in(x, y)$ and $r>0$ such that $[z-r, z+r] \subset(x, y)$ and $[z-r, z+r] \cap A_{*}=$ $\emptyset$. Since the sequence $\left(A_{k}\right)_{k \in \mathbb{N}}$ is descending, we see that there exists $k \in \mathbb{N}$ such that $[z-r, z+r] \cap A_{k}=\emptyset$. This implies that $f$ equals $f_{k}$ on $[z-r, z+r]$. Therefore, $f_{k}$ is constant on $[z-r, z+r]$, which contradicts that $f_{k}$ is strictly increasing as pointed out in assertion (iv) of Lemma 4.5.

Lemma 4.8. The function $f$ is a contraction.

Proof. Note that, by assertion (v) of Lemma 4.5, we have $|f(y)-f(x)|=$ $\lim _{k \rightarrow \infty}\left|f_{k}(y)-f_{k}(y)\right| \leq \frac{1}{2}|x-y|$ for all $x, y \in[0,1]$.

We finish this subsection with proving a property of $f$, which will be used later.

Lemma 4.9. If $x$ is an endpoint of an interval belonging to $\mathcal{I}_{k}$ for some $k \in \mathbb{N}$, then $f(x)=f_{k-1}(x)$.

Proof. Fix $k \in \mathbb{N}$ and an interval $[a, b] \in \mathcal{I}_{k}$. A trivial verification shows that for every $l \geq k$ the point $a$ is always a left endpoint of an interval from $\mathcal{I}_{l}$ and the point $b$ is always a right endpoint of an interval from $\mathcal{I}_{l}$. This jointly with assertion (ii) of Lemma 4.5 implies $f_{l}(a)=f_{k-1}(a)$ and $f_{l}(b)=f_{k-1}(b)$ for every $l \geq k$, and hence $f(a)=f_{k-1}(a)$ and $f(b)=f_{k-1}(b)$. 


\subsection{Definition of the IFS}

We define the announced IFS by taking $f$ and $g=f+\frac{2}{3}$.

By assertion (vi) of Lemma 4.5 we have

$$
f([0,1])=\left[0, \frac{1}{3}\right] \quad \text { and } \quad g([0,1])=\left[\frac{2}{3}, 1\right] .
$$

Moreover, from Lemmas 4.7 and 4.8 we see that our IFS consists of strictly increasing contractions.

If we show that its attractor is the set $A_{*}$, then the example will be complete.

Lemma 4.10. The set $A_{*}$ is the attractor of the IFS consisting of $f$ and $g$.

Proof. We first prove that

$$
A_{k+1}=f\left(A_{k}\right) \cup g\left(A_{k}\right)
$$

for every $k \in \mathbb{N}$.

Fix $k \in \mathbb{N}$. From assertion (viii) of Lemma 4.5 we conclude that $A_{k+1} \subset$ $f\left(A_{k}\right) \cup g\left(A_{k}\right)$. If we prove that $f\left(A_{k}\right) \cup g\left(A_{k}\right) \subset A_{k+1}$, the assertion follows.

Fix $x \in A_{k}$ and choose an interval $[a, b] \in \mathcal{I}_{k}$ such that $x \in[a, b]$. From Lemma 4.9 we get $f(a)=f_{k-1}(a)$ and $f(b)=f_{k-1}(b)$. Then using Lemma 4.7 and assertion (vii) of Lemma 4.5 we obtain

$$
f(x) \in[f(a), f(b)]=\left[f_{k-1}(a), f_{k-1}(b)\right] \subset \bigcup \mathcal{I}_{k+1}=A_{k+1} .
$$

Making also use of assertion (vi) of Lemma 4.5 and assertion (iv) of Lemma 4.2 we get

$$
g(x)=f(x)+\frac{2}{3} \in[f(a), f(b)]+\frac{2}{3} \subset \bigcup \mathcal{I}_{k+1}=A_{k+1},
$$

which proves (4.11).

In Lemma 4.3 we have recorded already that $A_{*}$ is nonempty and compact. According to Theorem 2.2 it remains to prove that $A_{*}=f\left(A_{*}\right) \cup g\left(A_{*}\right)$.

As $f$ and $g$ are strictly increasing as verified in the proof of Lemma 4.7, we have, using (4.9),

$$
\begin{aligned}
f\left(A_{*}\right) \cup g\left(A_{*}\right) & =\bigcap_{k \in \mathbb{N}} f\left(A_{k}\right) \cup \bigcap_{k \in \mathbb{N}} g\left(A_{k}\right) \\
& =\bigcap_{k \in \mathbb{N}}\left(f\left(A_{k}\right) \cup g\left(A_{k}\right)\right)=\bigcap_{k \in \mathbb{N}} A_{k+1}=A_{*} .
\end{aligned}
$$

The proof is complete. 


\section{Acknowledgements}

The research was supported by the University of Silesia Mathematics Department (Iterative Functional Equations and Real Analysis program). Furthermore, the research leading to these results has received funding from the European Research Council under the European Union's Seventh Framework Programme (FP/2007-2013)/ERC Grant Agreement No. 291497 while the second author was a research fellow at the University of Warwick.

Open Access. This article is distributed under the terms of the Creative Commons Attribution 4.0 International License (http://creativecommons.org/licenses/ by/4.0/), which permits unrestricted use, distribution, and reproduction in any medium, provided you give appropriate credit to the original author(s) and the source, provide a link to the Creative Commons license, and indicate if changes were made.

\section{References}

[1] Calabrò, F., Corbo Esposito, A., Mantica, G., Radice, T.: Refinable functions, functionals, and iterated function systems. Appl. Math. Comput. 272(part 1), 199-207 (2016)

[2] Crovisier, S., Rams, M.: IFS attractors and Cantor sets. Topol. Appl. 153(11), 1849-1859 (2006)

[3] D'Aniello, E.: Non-self-similar sets in $[0,1]^{N}$ of arbitrary dimension. J. Math. Anal. Appl. 456(2), 1123-1128 (2017)

[4] D'Aniello, E., Steele, T.H.: Attractors for iterated function schemes on $[0,1]^{N}$ are exceptional. J. Math. Anal. Appl. 424(1), 537-541 (2015)

[5] D'Aniello, E., Steele, T.H.: Attractors for iterated function systems. J. Fractal Geom. 3(2), 95-117 (2016)

[6] Falconer, K.: Fractal Geometry. Mathematical Foundations and Applications, 2nd edn. Wiley, Hoboken (2003)

[7] Matkowski, J.: Remark on BV-solutions of a functional equation connected with invariant measures. Aequ. Math. 29(2-3), 210-213 (1985)

[8] Morawiec, J.: On $L^{1}$-solutions of a two-direction refinement equation. J. Math. Anal. Appl. 354(2), 648-656 (2009)

[9] Morawiec, J., Zürcher, T.: On a problem of Janusz Matkowski and Jacek Wesołowski. Aequ. Math. (2018). https://doi.org/10.1007/s00010-018-0556-5

[10] Peres, Y., Solomyak, B.: Self-similar measures and intersections of Cantor sets. Trans. Am. Math. Soc. 350(10), 4065-4087 (1998) 
Janusz Morawiec and Thomas Zürcher

Instytut Matematyki

Uniwersytet Śląski

Bankowa 14

40-007 Katowice

Poland

e-mail: morawiec@math.us.edu.pl

Thomas Zürcher

e-mail: thomas.zurcher@us.edu.pl

Received: December 5, 2017.

Accepted: April 13, 2018. 